\title{
EFFECT OF CONTEXT AND EFFERENCE COPY ON VISUAL STRAIGHT AHEAD
}

\author{
Bruce Bridgeman and Judith Ann Graziano \\ Program in Experimental Psychology, Clark Kerr Hall, University of California, \\ Santa Cruz, CA 95064, U.S.A.
}

(Received 29 July 1988; in revised form 24 April 1989)

\begin{abstract}
Bias in efferent commands to the eye changes the apparent straight ahead direction in an unstructured visual field, but has little effect in a normal visual environment. Naive subjects set a visible marker to appear straight ahead under monocular viewing conditions and while pressing on the viewing eye. Three background conditions were used: a naturalistic landscape photograph, a blank field, and a repeating checkerboard texture that provides strong contours but no information about visual direction. Effect of eyepress on straight-ahead judgments was small but significant with the landscape background, and larger with the blank field; the checkerboard texture yielded a bias halfway between the magnitudes of bias in the other two conditions. A visual capture theory predicts that the textured field should work like a blank one, while an oculomotor theory predicts that it should work like a natural one. Interpreted in this context, the results show the two theories to be about equally important in judging straight ahead. A second experiment with experienced observers and moving backgrounds gave the same result.
\end{abstract}

Efference copy Visual capture Straight ahead Oculomotor Eyepress Eye movements

\section{INTRODUCTION}

Determination of visual direction relative to the head requires information about both the position of the image on the retina and the position of the eyes in the head. The common assumption has been that these two sources of information add algebraically to give the position of an object relative to the head (Helmholtz, 1867).

In fact the interaction is more complex: the algebraic summation of image-in-eye and eyein-head information determines perception of direction only when visual context is absent. This result has been found with two methodsparalysis and ocular displacement (eyepress). Using the first method, partial paralysis with a synthetic curare, Matin, Picoult, Stevens, Edwards, Young and MacArthur (1980, 1982) found that perceived position of a small luminous target in darkness is biased in the direction of attempted gaze. In the structured visual field of a normal laboratory environment, however, abnormal efference had no noticeable effect on judgment of visual direction-the perceptual bias in position occurred only if the target was presented in darkness.

A similar result has been obtained with the ocular displacement method, using a sustained press on the eye to dissociate eye position from oculomotor efference (Bridgeman \& Stark, 1981; Stark \& Bridgeman, 1983; Hershberger, 1984; Bridgeman, 1986). Because the method is also used in the present paper, the experiments and theory that validate and develop the method will be reviewed in some detail. The ocular displacement method is executed in a monocularly viewing subject with the head mechanically restrained. As the subject slowly begins to press with the finger on the outer canthus of the viewing eye, while visually fixating a point, the following events take place. At first the finger generates a rotational force that begins to rotate the eye medially. As the right eye is pressed, for example, it is rotated to the left. As soon as the eye begins to move, however, the oculomotor systems that maintain visual fixation are stimulated to perform corrective innervations. The lateral rectus muscle rotates the eye to the right, cancelling the retinal slip and maintaining the position of the image on the retina. As the pressure of the finger increases, the oculomotor compensation increases proportionately. Since the extraocular muscles are very strong compared to their loads, they succeed in maintaining image position on the retina.

Stability of image position on the retina during gradual eyepress has been demonstrated 
experimentally with two methods. A psychophysical demonstration used a red-green color discrimination in very small ( $9 \mathrm{~min}$ arc square) targets. The discrimination could be performed reliably only in foveal vision: performance for targets $3 \mathrm{deg}$ from the foveal center was random. Discrimination remained unchanged, however, during unrestrained fixation, during eyepress, and during the dynamic phase of a gradual eyepress when the subjects were experiencing apparent motion of the target (Stark \& Bridgeman, 1983).

A second method determines fixation position directly, using a binocular search coil technique to measure the orientations of both eyes during ocular displacement. With this recording method, rotational position of an eye can be distinguished from lateral translation of the eye in the orbit. During a gradual eyepress the rotational orientation of the viewing, pressed eye remained constant, while the occluded eye rotated under its occluder in the direction opposite the rotational force applied by the finger (Ilg, Bridgeman \& Hoffman, 1989). Thus the occluded eye revealed the oculomotor innervation that was being exerted on the viewing eye as well, according to Hering's law of equal innervation (Hering, 1868). (The recordings showed that active compensation for the effects of eyepress occurs only with very slow dynamics; if the eye is pressed and released alternately at a rapid rate, the pressed eye rotates and the occluded eye does not.)

The experiments show that a gradual press on the eye biases oculomotor efference without changing the projections of objects on the retina. An apparent shift of the visual world in the direction opposite the direction of the press results, though retinal image position remains constant. As the finger presses harder and harder on the eye, oculomotor gaze stabilization systems resist the eye press more and more strongly, resulting in an increasing deviation of oculomotor efference from actual eye position. The change in efference can be measured directly in the occluded fellow eye.

This conclusion depends upon stability of vergence position. A change in vergence, because it would move the occluded eye without affecting the position of the viewing, pressed eye, might be misinterpreted as a change in the efference copy of the signal to the viewing eye. The most stringent test of this possibility is to record the occluded eye in complete darkness, where no target is present to stabilize accomodation state. Under these conditions, the occluded eye remains quite stable, showing no noticeable effects of vergence change in the short term (Bridgeman \& Delgado, 1984). In those experiments and in the present study we used an oculomotor trick to minimize vergence: since the eyes tend to diverge with upward gaze positions and remain in stable vergence positions (Heuer, Dunkel-Abels, Brüwer, Kröger, Römer \& Wischmeier, 1988), eyes were deviated upward. The limit on divergence beyond parallel lines of sight in normal subjects reduces vergence variability because of a "ceiling" effect.

Hence apparent motion of the world results not from a movement of the image on the retina, as was believed by Descartes, Purkinje and Helmholtz, but from a change in efference to the pressed eye. The actual motion of the image on the retina is small, and changes in magnitude and direction as the oculomotor stabilization mechanisms are activated to reduce the motion. The perception of motion of the visual world when the eye is pressed cannot originate from this retinal image motion-rather, the efferent command itself must be monitored centrally to inform the visual system of the intended movements of the eye. This internal monitor has been called the efference copy (von Holst \& Mittelstaedt, 1950) or the corollary discharge (Sperry, 1950). If the efference copy is continuously changing, and the image position on the retina is relatively stable, the visual system concludes that the object of vision is moving.

Under normal conditions, subjects estimating straight-ahead location set a reference spot to the same location whether the viewing eye is pressed or not. In darkness, however, efference copy (measured by the deviation of the occluded fellow eye) determines the position estimates (Bridgeman \& Stark, 1981; Stark \& Bridgeman, 1983). Perceived deviation of the straight-ahead direction can be predicted quantitatively from the bias in oculomotor efference. This result is in agreement with that of Matin et al. (1982).

Two theories have emerged to explain dominance of retinal information in structured fields: the visual capture theory and the oculomotor theory. The visual capture theory asserts that the visual system rejects efferent information about eye position in favor of retinal information. The structure of the visual field, rather than its location on the retina and the retina's position in the head, determines perceived location. Since retinal information captures efferent information, rather than auditory or other 
sources, this is a special case of visual capture which has been called visual capture of Matin (VCM) (Stark \& Bridgeman, 1983).

The oculomotor theory proposes that an optokinetic system contributes to oculomotor posture, stabilizing the position of the image on the retina with a negative feedback mechanism that does not involve perception. The mechanism concerns only control of fixation, and is distinct from the perceptual stabilizations of space constancy. Normally, this mechanism stabilizes the position of the image on the retinas (with enough inaccuracy to prevent image fading); in a rotating drum, the system generates optokinetic nystagmus (OKN).

The theory assumes the interaction of several sources of oculomotor innervation for gaze stabilization. Unregistered optokinetic innervation stabilizes the retinal image as a whole, preventing movement of the entire retinal image by generating slow eye movements to compensate for image drift. Pursuit innervation performs a complementary function, stabilizing the position of the foveal image. Both types of innervation are active simultaneously, even when the eye is not moving, and they can be antagonistic under some visual conditions. When conflict arises, pursuit can dominate optokinetic stabilization efforts. Maintaining steady fixation during induced target motion, for instance, requires a pursuit signal that counteracts the tendency of optokinetic innervation to move the eye in the direction of the background (Post, Shupert \& Leibowitz, 1984).

Bridgeman (1986) extended this analysis to static conditions, noting that in a structured visual field the optokinetic stabilization system could maintain fixation of a target despite changes in efference during eyepress. Because the optokinetic system would compensate image drift, the pursuit system would not be stimulated significantly, and therefore perception would remain unaffected as well. Applied to the problem of determining straight ahead during eyepress, this analysis maintains that the efference copy need not be suppressed as the visual capture theory requires. The effects of altered efference are countered by compensatory changes in the optokinetic stabilization system, without affecting perception.

Since oculomotor stabilization is effective only in a structured visual field, the perceived straight ahead direction will change very little in a structured field. With only a single point in darkness, however, the optokinetic system cannot maintain fixation on the target, and an offset of efferent signals will require additional compensatory pursuit innervation. This pursuit innervation will be perceived as a changed position of the target.

In this study, the roles of these two hypothesized mechanisms, visual capture and oculomotor stabilization, are compared in an attempt to discover how visual straight ahead is determined. In order to differentiate the oculomotor and the visual capture theories, we needed an experimental condition in which the two theories would make different predictions. Such a condition exists in the difference between the structure of the visual information required by the oculomotor system and the visual capture mechanism respectively. Visual capture depends upon the structure of the visual field. Perspective, location and size of identifiable objects, texture gradients, etc., comprise visual structure. If the visual capture theory best explains straightahead judgments, stabilization will occur only in structured environments; an environment with texture but no structure (i.e. a uniform texture) will be functionally equivalent to a blank field and produce no stabilization. The oculomotor theory, however, relies solely upon the presence of texture: contrasting elements in the visual array. If the oculomotor theory is correct, stabilization of straight ahead will occur in both textured and structured visual fields; only the absence of a visual field should result in destabilization.

There are two ways to design an experiment contrasting straight-ahead estimations with varying backgrounds: one is to project a target point against a background and ask subjects to move the point to the apparent straight ahead. With this method the background position remains constant, but the presence of the point changes the stimulus configuration as a function of the change in apparent straight-ahead direction. The second method is to move the background itself until a feature of the background pattern appears to be straight ahead. With this method the independent variable (background) is confounded with the dependent variable (position setting), but the stimulus configuration remains constant. We have done the experiment both ways, a full-scale study with an adjustable point and fixed background array, and a smaller confirmation study with an adjustable background. 


\section{EXPERIMENT I}

\section{Method}

Apparatus. A white screen of uniform curvature, $170 \mathrm{deg}$ wide by $50 \mathrm{deg}$ high, was positioned $58 \mathrm{~cm}$ in front of an observer seated at the center of curvature of the screen. A white " $X$ " originated from a slide projector and reflected into a small galvanometer mirror whose angle of reflection on the screen was controlled by adjustment of a potentiometer. The mirror was located just under the subject's head, with its axis of rotation near the center of curvature of the screen. The $\mathrm{X}$ disappeared at the end of its range, about $20 \mathrm{deg}$ to the right of the objective centerline of the apparatus. A simple circuit allowed the subject to move the projected image horizontally, with a hand-held knob. The knob (attached to the potentiometer) was radially symmetrical so that it could not provide information about exocentric position of the image on the screen. Knob manipulation allowed a subject to indicate perceived straightahead direction. An analog voltage from the circuit was sent to the A/D converter of a laboratory computer so that knob position could be recorded.

To stabilize the subject's head a bite bar was secured above the table at a height approximating normal mouth position of the seated subject. A bite bar of dental wax was moulded for each subject. Immediately anterior to the bar a horizontal black baffle obstructed the bottom portion of the screen and projection apparatus. This arrangement is adequate to stabilize the head during the mild self-applied presses on the eye.

Elevated behind the subject was a projector equipped with a computer-controlled shutter and galvanometer mirror. Slide 1 depicted an outdoor landscape scene. Slide 2 was composed of a uniform black and white checkerboard, oriented diagonally. Horizontal distance between the centers of the checks ("diamonds") was $3.6 \mathrm{deg}$, so that the fundamental spatial frequency of the pattern was $0.28 \mathrm{c} / \mathrm{deg}$. Slide 3 was an opaque slide generating a blank field.

Eye movements were monitored with an infrared photoelectric method (modified from Bahill et al., 1975). Two photocells and an infrared LED were mounted on a metal bar attached to the same frame that supported the bite bar. A 3-dimensional stage allowed adjustment of the photocell array with thumbscrews. Eye movements were calibrated by asking a subject to fixate targets centered in the field, and $5 \mathrm{deg}$ left and right at the same vertical gaze angle as the $\mathrm{X}$-shaped target that was used in experimental trials. The apparatus was calibrated to achieve equal gain on the two sides.

The infrared method has the advantages that nearly the entire visual field is unrestricted, and the apparatus does not contact the eye. The technique is sensitive to translations of the eye, however. In the present experiments, artifactual deviations in the eye movement record due to head movement with respect to the recording apparatus would be revealed as apparent zerolatency eye movements of the occluded right eye to the right, coinciding with the eyepress. Such movements were never observed. Instead, recordings showed that the occluded eye deviated to the left after a normal latency of about $150 \mathrm{msec}$, matching the direction of the apparent deviation experienced by the subjects. Published recordings from our laboratory (Bridgeman \& Delgado, 1984) show that no deviation of the fellow eye occurs during eyepress in complete darkness if the head is secured in a bite bar.

Procedure. Ten naive subjects participated in the study. One of them was disqualified after a previously undiagnosed latent nystagmus was discovered during the calibration procedure for the eye movement recordings. Data are reported for the remaining nine subjects. After practice of the eyepress maneuver, subjects were qualified as proficient in effecting a good horizontal deviation of oculomotor efference by measuring deviation of the occluded right eye during press of the left eye. Criterion for effective eyepress was a deviation of at least $2 \mathrm{deg}$ in the occluded eye on two out of 3 trials; all subjects met this criterion after the practice period. Gaze was first directed to the angle to be used in the experiments, near the straight ahead direction and elevated about $35 \mathrm{deg}$, an angle that aids in achieving a horizontal apparent motion with eyepress, minimizing other apparent deviations. Horizontal was defined in terms of the visual world rather than eye coordinates; subjects attempted to achieve a deviation that follows a horizontal straight line such as the intersection of a wall with a ceiling. At this gaze angle, subjects manipulated the direction and location of the press on the eye with their fingers until they reached the criterion.

Straight ahead was defined for the subjects in terms of an imaginary plane bisecting the subject's head on its axis of symmetry. Subjects 
were instructed to find the straight-ahead position by imagining themselves moving straight ahead into the screen, without deviating left or right, then move the white " $X$ " to that spot.

At the start of the experiment, subjects were seated before the screen and informed that they would be shown one of three slides. For each slide, subjects were to find straight-ahead twice, once without eyepress (control trial), and once with eyepress to the left eye (experimental trial); the right eye was occluded throughout the session. In the control portion of each trial pair, subjects began with illuminated $X$ in its occluded off-field position, moved the $X$ to straight-ahead by manipulating the knob, and returned the $\mathrm{X}$ off-field. In the experimental portion, the eye was pressed in the dark before the slide appeared, and was held while the slide was presented and straight-ahead located. After the subject's response was recorded, the eye was released and the $X$ was again moved off-field.

A total of 30 trial pairs was run: 6 trial pairs for each of the 3 slides, randomly mixed with an additional 6 pairs of both the landscape and diamond pattern which incorporated horizontal physical deviation of the slide position between the control and experimental trials. Range of deviation varied from 2 to $6 \mathrm{deg}$; subjects were informed that the patterns would sometimes appear shifted, and that this was not an illusion. Only the 3 trial types without deviation were analyzed, however. The two deviation conditions served to discourage subjects from using landmarks on the image to make the straightahead judgement. Slide order was randomized, as was slide deviation in the two types of deviation trials.

Global regressions or analyses of variance are not appropriate methods of analysis because background condition is a nominal variable. Analysis thus consisted of planned comparisons to test the above hypotheses. For each subject, $t$-tests were also performed to determine the significance of the differences between the control and the eyepress conditions for each of the three trial types.

\section{Results}

Eight of the nine subjects showed statistically significant differences in straight-ahead settings between the eyepress and control conditions for at least one of the backgrounds. Seven of the nine showed differences between eyepress and control with the blank background at $P<0.05$
(4 at $P<0.01$ ); five showed differences with the diamond texture background ( 4 at $P<0.01$ ), and four showed differences with the landscape background (all at $P<0.01$ ).

If the three conditions are arranged in order of increasing strength of effect (landscape, diamonds, blank), most subjects who showed effects in one condition also showed effects in the successive conditions. Subjects who showed significant effects for the landscape also showed significant effects for the diamonds and the blank; those who showed effects for the diamonds also showed significance for the blank.

There were two exceptions to this pattern: one subject showed significant effects in the diamond condition but not in the others, and another subject showed effects in the landscape and blank but not the diamonds. In the latter subject, however, the largest mean difference between eyepress and control trials was in the diamond condition, showing that the lack of significance is due entirely to larger variability in this condition. This subject also showed the smallest amount of mean difference between the conditions of the nine subjects in the sample. In general, significance in the landscape condition implied significance in the diamonds and the blank; significance in the diamonds implied significance in the blank.

The differences between responses with the various backgrounds werc further analyzed with between-subjects tests on the magnitudes of differences between the conditions, with each subject contributing one mean deviation in each condition. For the landscape there was a small $(0.95 \mathrm{deg})$, marginally significant difference between eyepress and control settings $t_{8}=2.31$, $P=0.0497$ ). For the blank field the mean difference in straight-ahead settings was $2.875 \mathrm{deg}$, a highly significant figure $\left(t_{8}=6.56, P=0.00018\right)$ (Fig. 1). All subjects showed a greater deviation in the blank condition than in the landscape condition. The deviations were in the predicted directions, i.e. apparent deviation toward the left when the left eye is pressed.

The key result for testing the two theories of straight-ahead, the straight-ahead deviation with the diamond texture background, was almost precisely halfway between the landscape and the blank settings: mean difference was $1.86 \mathrm{deg}\left(t_{8}=4.56, P=0.0018\right)$. The oculomotor theory predicts that this value should have been close to the deviation in the landscape, $0.95 \mathrm{deg}$; the visual capture theory, in contrast, predicts that the value should have 
been close to the deviation with no background, $2.875 \mathrm{deg}$.

The mean deviation of the right (occluded) eye was $3.83 \mathrm{deg}$, not significantly different from the psychophysical deviation of straight ahead in the blank condition $\left(t_{8}=1.887, P=0.153\right)$ (Fig. 2). Eye deviations were toward the left, corresponding to the direction of apparent motion. Thus performance in the blank field followed the changed efference to the eye, while the deviations with backgrounds were significantly smaller than this (eye vs diamonds, $t_{8}=2.63, P=0.030$; eye vs landscape, $t_{8}=3.834, P=0.0050$ ).

\section{EXPERIMENT II}

\section{Method}

This mini-experiment was intended to check the results of expt I with a 1-channel stimulus configuration. Screen and viewing conditions were the same, but all of the stimuli were different than those used in expt I. Slide 1 was an outdoor mountain landscape, with a castle at the center that was used as a target spot to indicate the straight ahead. A different scene was used to test whether idiosyncracies in the scene of expt I might have led to anomalous results. Slide 2 was a random-dot texture, with a larger center dot that the subject could use as a target spot. This is a nonrepeating texture, in contrast to the repeating texture of expt $I$. Slide 3 was a single green " $X$ " on a blank field.

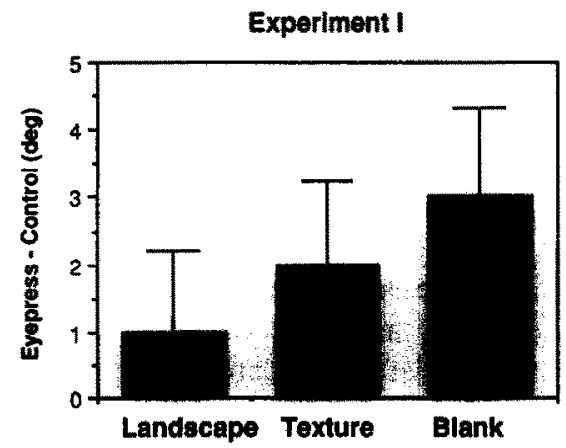

Fig. 1. Differences in straight-ahead judgments in eyepress vs control trials, under three fixed background patterns. Each bar summarizes data from a total of 45 trial pairs (one experimental judgment, one control) in 9 subjects. Error bars given the between-subjects standard deviation. The "texture" condition, containing contour but no structure useful in determining visual direction, gives results intermediate between the naturalistic landscape and the blank conditions. All differences between heights of bars are statistically significant.

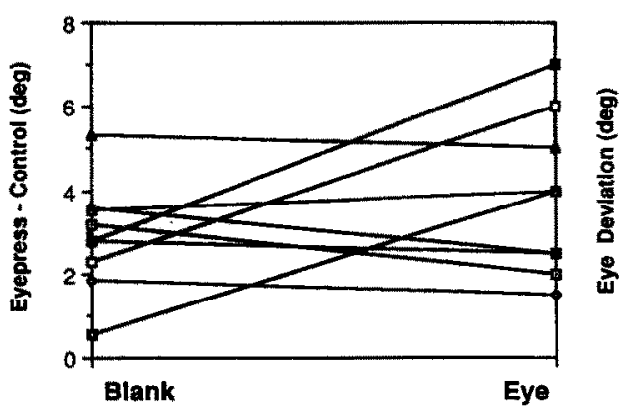

Fig. 2. Relation between deviations in straight-ahead (left) and objective deviation of the occluded fellow eye (right) for each of the 9 subjects. There is no statistically signifcant difference between the two distributions. Removing the 3 subjects with eye deviations that are much larger than the psychophysical deviations does not change the results in Fig. 1: deviations in the texture condition are still intermediate between the landscape and the blank conditions.

The mirror in the background projector's light path was now controlled by the subject, using the potentiometer circuit of expt $\mathrm{I}$.

Instructions were similar to those in expt I; subjects were asked to adjust the pattern position until they felt that they could meet the target spot if they moved straight ahead along the median plane, without deviating right or left. Each of three subjects was run for 20 trials with each slide in a blocked design, but because the subjects complained about disorientation and fatigue with so many trials, only the first 5 trials of each condition were scored. Some of the disorientation was caused by an upward autokinesis that was noted mostly in the single- $x$ field. The $x$ seemed to drift upward after a sustained elevation of gaze. All subjects were experienced psychophysical observers; none of them had participated in expt I. Thus relationships between the results of expts I and II are based on between-subjects comparisons.

\section{Results}

Despite the many differences in display, method and subjects, the pattern of results was similar to that in expt I. With the landscape, deviations between eyepress and control trials were not significant for 2 of the three subjects; with the random-dot pattern, deviations were significant for all three subjects $\left(t_{4}=4.71\right.$, $P=0.009 ; \quad t_{4}=6.93, \quad P=0.002 ; \quad t_{4}=4.99$, $P=0.0075$ ). Deviations between eyepress and control trials were also significant for all subjects with the blank field $\left(t_{4}=4.49, P=0.0109\right.$; $\left.t_{4}=6.26, P=0.003 ; t_{4}=9.93, P=0.0006\right)$. The mean deviation was $3.6 \mathrm{deg}$ for the landscape 
and 9.04 deg for the blank field. All subjects showed a greater deviation in the blank condition than in the landscape condition.

Again, the mean deviation for the textured field was almost exactly intermediate between these two extremes, at $6.58 \mathrm{deg}$ (Fig. 3). Reflecting the accuracy of performance, subjects felt that the task was easiest with the landscape, harder with the random dots, and harder yet with the single $x$.

All deviation values are larger than those in expt I, probably because the experienced observers achieved larger deviations of the fellow eye with eyepress (mean eye deviation $=$ $5.33 \mathrm{deg}$ ). Again, the objectively measured eye deviation did not differ significantly from the psychophysical deviations in the blank condition $\left(t_{2}=3.21, P=0.085\right)$.

\section{GENERAL DISCUSSION}

In both experiments, the pattern containing texture but no meaningful structure showed a stabilization intermediate between the stabilizations for the fully structured field and for the single point. Interpreted in terms of the two theories introduced above, the intermediate value means that both influences, oculomotor and visual capture, contribute about equally to visual straight ahead.

Neither theory alone can account for all of the results. The visual capture theory accounts for the larger deviation with a textured field than with a fully structured landscape, but it cannot account for the fact that the deviation of the textured field is smaller than that for a single point. It also cannot explain the small but significant deviations found even in the landscape condition.

The oculomotor theory accounts for the smaller deviation of straight ahead with the textured field than with the single point, but it cannot explain why the differences in judgements for the textured field should be greater than those for the landscape. Since efference is always active according to the oculomotor theory, even in the landscape condition, this theory can explain the small deviations found with the landscape.

The efference copy mechanism (von Holst \& Mittelstaedt, 1950) must have a gain of less than 1 to remain stable. Subsequent quantitative work has provided estimates of the gain of the efference copy in large fields; Dichgans, Körner and Voigt (1969) found a subjective velocity

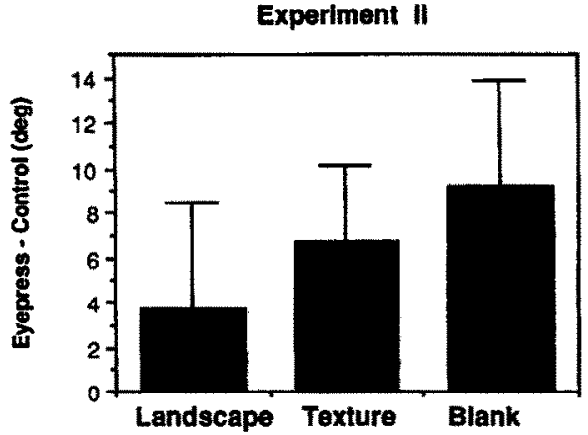

Fig. 3. Differences in straight-ahead judgments in eyepress vs control trials, under three background conditions, with backgrounds that move left and right under control of the subject. Display format as in Fig. 1. Landscape and texture patterns are different from those in expt $I$.

1.6-1.66 times larger from retinal motion than from tracking of a moving texture; taking the reciprocal of these values yields a gain of 0.602 0.625 for the efference copy. These values are close to older estimates from more qualitative experiments in the psychophysical literature.

The oculomotor results are consistent with the conclusion of Yasui and Young (1975) that efference copy gains are less than 1; they also point out that gain adaptation makes these values vary according to stimulus conditions. In studies of smooth pursuit tracking they find evidence that the efference copy is active even under normal closed-loop conditions, not only during saccades. The present results also imply substantial individual differences in efference copy gain.

In sum, the present experiments can be explained only by combining the mechanisms proposed by both theories, VCM and oculomotor, operating in parallel. Neither theory alone can account for all of the data. Since the two theories are not mutually contradictory, the most reasonable conclusion at present is that both mechanisms are working simultaneously to arrive at judgments of straight ahead. Efferent information is weighted along with VCM, and is not suppressed. Incomplete suppression of oculomotor efferent signals in the landscape condition indicates that an oculomotor mechanism is operating even when visual capture information is available.

This idea is consistent with Shebilske's (1986) "ecological efference mediation theory", a perspective that allows multiple sources of information to contribute simultaneously to spatial judgments. It is formalized in De Graaf and Wertheim's (1988) suggestion that motion is 
evaluated not against efference alone, but against a "reference signal" consisting of efferent information, afferent vestibular signals, and afferent retinal optokinetic information.

The simultaneous application of several mechanisms to a single problem is common in biological information processing. An example is light adaptation: many mechanisms, at different levels, share a common goal. Pupil, photopigment chemistry, and neural interactions all contribute to light adaptation. A similar approach is taken in Ramachandran's (1987) "bag of tricks" theory of perception with the idea that many simple algorithms replace a few complex and general ones in visual function. This paper suggests that egocentric localization should include at least two types of mechanisms, visual capture and oculomotor, in its "bag of tricks".

\section{REFERENCES}

Bahill, A. T., Clark, M. E. \& Stark, L. (1975). Dynamic overshoot in saccadic eye movements is caused by neurological control signal reversals. Experimental Neurology, $48,95-112$.

Bridgeman, B. (1986). Multiple sources of outflow in processing spatial information. Acta Psychologica, 63, 35-48.

Bridgeman, B. \& Delgado, D. (1984). Sensory effects of eyepress are due to efference. Perception and Psychophysics, 36, 482-484.

Bridgeman, B. \& Stark, L. (1981). Efferent copy and visual direction. Investigative Ophthalmology and Visual Science (Suppl.), 20, 55.

De Graaf, B. \& Wertheim, A. (1988). The perception of object motion during smooth pursuit eye movements: Adjacency is not a factor contributing to the Filehne illusion. Vision Research, 28, 497-502.

Dichgans, J., Körner F. and Voigt K. (1969). Vergleichende Skalierung des afferenten unde efferenten Bewegungssehens beim Menschen: Lineare Funktionen mit verschiedener Anstiegssteilheit. Psychologische Forschung, 32, 277-295.
Helmholtz, H. (1867). Handbuch der physiologischen Optik (Vol. 3). Leipzig: Voss.

Hering, E. (1868). Die Lehre vom Binokularen Sehen. Leipzig: Engelman. (Translation edited by Bridgeman, B. \& Stark, L. (1977). The Theory of Binocular Vision. New York: Plenum Press.)

Hershberger, W. (1984). Impressions of visual direction from extraocular afference. Perception and Psychophysics, $35,400-401$.

Heuer, H., Dunkel-Abels, G., Brüwer, M., Kröger, H., Römer, T. \& Wischmeier, E. (1988). The effects of sustained vertical gaze deviation on the resting state of the vergence system. Vision Research, 28, 1337-1344.

Ilg, U., Bridgeman, B. \& Hoffman, K.-P. (1989). Influence of mechanical disturbance on oculomotor behavior. Vision Research, 29, 545-551.

Matin, L., Picoult, E., Stevens, J. K., Edwards Jr, M. W., Young, D. \& MacArthur, R. (1980). Visual context dependent mislocalizations under curare-induced partial paralysis of the extraocular muscles. Investigative Ophthalmology and Visual Science, (Suppl.), 19, 81.

Matin, L., Picoult, E., Stevens, J. K., Edwards Jr, M. W., Young, E. \& MacArthur, R. (1982). Oculoparalytic illusion: Visual-field dependent spatial mislocations by humans partially paralyzed with curare. Science, New York, 216, 198201.

Post, R. B., Shupert, C. \& Leibowitz, H. W. (1984). Implications of OKN suppression by smooth pursuit for induced motion. Perception and Psychophysics, 36, 493-498.

Ramachandran, V. (1987). Shape-from-shading and illusory contours as textons. Paper read at Psychonomic Society 28th Annual Meeting, 7 November, 1987.

Shebilske, W. L. (1986). Baseball batters support an ecological efference mediation theory of natural event perception. Acta Psychologica, 63, 117-131.

Sperry, R. (1950). Neural basis of the spontaneous optokinetic response produced by visual inversion. Journal of Comparative and Physiological Psychology, 43, 482-489.

Stark, L. \& Bridgeman, B. (1983). Role of corollary discharge in space constancy. Perception and Psychophysics, $34,371-380$.

Von Holst, E. \& Mittelstaedt, H. (1950). Das Reafferenzprinzip (wechselwirkungen zwischen Zentralnervensystem und Peripherie). Naturwissenschaften, 37, 464-476.

Yasui, S. \& Young, L. R. (1975). Perceived visual motion as effective stimulus to pursuit eve movement system. Science, New York, 190, 906-908. 